\title{
Alleviation of Salinity-Induced Oxidative Stress, Improvement in Growth, Physiology and Mineral Nutrition of Canola (Brassica napus L.) through Calcium-Fortified Composted Animal Manure
}

\author{
Muhammad Naveed ${ }^{1, *}$, Haroon Sajid ${ }^{1}$, Adnan Mustafa ${ }^{1,2}\left(\mathbb{D}\right.$, Bushra Niamat ${ }^{1}$, Zulfiqar Ahmad ${ }^{1}$, \\ Muhammad Yaseen ${ }^{1}$, Muhammad Kamran ${ }^{3}$ (D), Munazza Rafique ${ }^{4}$, Sunny Ahmar ${ }^{5}$ and \\ Jen-Tsung Chen ${ }^{6, *}$ iD \\ 1 Institute of Soil and Environmental Sciences, University of Agriculture, Faisalabad 38040, Pakistan; \\ eagle.ka4u786@yahoo.com (H.S.); adnanmustafa780@gmail.com (A.M.); bushraniamat77@gmail.com (B.N.); \\ zulfiqar1409@gmail.com (Z.A.); dr.yaseen@gmail.com (M.Y.) \\ 2 National Engineering Laboratory for Improving Quality of Arable Land, Institute of Agricultural Resources \\ and Regional Planning, Chinese Academy of Agricultural Sciences, Beijing 100081, China \\ 3 Key Laboratory of Arable Land Conservation (Middle and Lower Reaches of Yangtze River), Ministry of \\ Agriculture, College of Resources and Environment, Huazhong Agricultural University, Wuhan 430070, \\ China; kamiagrarian763@gmail.com \\ 4 Soil Bacteriology Section, Agri. Biotechnology Research Institute, AARI, Faisalabad 38000, Pakistan; \\ munazzaaari@gmail.com \\ 5 National Key Laboratory of Crop Improvement Genetics, College of Plant Science and Technology, \\ Huazhong Agricultural University, Wuhan 430070, China; sunny.ahmar@yahoo.com \\ 6 Department of Life Sciences, National University of Kaohsiung, Kaohsiung 811, Taiwan \\ * Correspondence: muhammad.naveed@uaf.edu.pk (M.N.); jentsung@nuk.edu.tw (J.-T.C.)
}

Received: 24 December 2019; Accepted: 21 January 2020; Published: 23 January 2020

\begin{abstract}
Salinity stress is one of the serious restrictive issues for optimum crop production in arid to semi-arid areas. Application of organic amendments have shown positive effects on crop growth and yield under such scenario. The present study was conducted to estimate the potential of calcium-fortified composted animal manure (Ca-FCM) to enhance growth and yield of canola under saline soil conditions. Salt affected soils with various electrical conductivity (EC) levels (original 1.5, 5 , and $10 \mathrm{dS} \mathrm{m}^{-1}$ ) were developed via spiking the soil with sodium chloride $(\mathrm{NaCl})$ salt. The results reveal that soil salinity reduced the growth, physiological, yield, and nutritional parameters of canola. However, application of $3 \%$ calcium-fortified composted manure significantly enhanced the growth and yield parameters at all EC levels as compared to control. Plant physiological parameters such as photosynthetic rate, relative chlorophyll contents (SPAD value), and relative water content were also increased with the application of $3 \% \mathrm{Ca}-\mathrm{FCM}$ at all EC levels in comparison to control. Application of 3\% Ca-FCM also mediated the antioxidant enzymes activities at all EC levels in comparison to control. Moreover, application of 3\% Ca-FCM caused maximum increase in nitrogen, phosphorus, and potassium concentrations in shoot at all EC levels. Conversely, application of $3 \%$ Ca-FCM showed maximum decrease in $\mathrm{Na}^{+} / \mathrm{K}^{+}$ratio in leaf up to $83.33 \%, 77.78 \%$, and $71.43 \%$ at EC levels $1.5,5$, and $10 \mathrm{dS} \mathrm{m}^{-1}$, respectively, as compared to control. It was concluded that application of calcium-fortified composted animal manure (Ca-FCM) could be an efficient method for improving growth, yield, physiological, and nutritional parameters of canola through mediation of antioxidant defense machinery under saline soil conditions.
\end{abstract}

Keywords: salinity; manure; yield; Ca-fortification; antioxidants; canola 


\section{Introduction}

Abiotic environmental stresses are notorious issues in agricultural production causing decline in crop yields around the globe [1,2]. Among abiotic stresses, soil salinity due to accumulation of soluble salts (mainly $\mathrm{NaCl}$ ) in arable soils is a key hazard to sustainable crop production especially in arid and semi-arid regions. Higher levels of $\mathrm{Na}^{+}$salts alter physico-chemical properties of the soils such as decreased soil porosity, water movement, and soil aeration which negatively affect uptake of essential plant nutrients and access to water by plants [3,4]. Such salt-affected soils are generally distributed throughout the world, comprising $20 \%$ of the world's cultivated land [5] and $50 \%$ of croplands are being exacerbated by varying degrees of salt accumulation [6]. Moreover, $20 \%$ of the world's arable land is currently exposed to salt stress and this will continue to increase up to $50 \%$ by 2050 [7]. Conversion of arable land to degraded land and crop yield losses are attributed to high salinity levels [8]. These losses are of significant considerations for some developing countries such as Pakistan where the economy mostly relies on agriculture [9]. In Pakistan, according to an estimate, about 6.63 mha lands are affected by salinity [10], amounting to one-third of the world productive land depleted by salt stress. Area of agricultural arable land decreases due to salt stress, which might seriously distress the relative extents of food supply and demand in the future.

Saline soils are characterized by high absorptions of soluble salts and low uptake of essential mineral nutrients such as nitrogen [11]. High concentration of salts in soils limits seed germination and root proliferation and obstructs uptake of water and nutrients to plants, which further reduces photosynthetic activity and vegetative growth of plants [12-14]. Alteration in osmotic and matric potential of soil solution occurs due to salt stress, which adversely affects microbial communities in soil and their activities, which are otherwise important for nutrient cycling in the environment $[15,16]$. Furthermore, osmotic and ion-specific effects caused by salinity create imbalances in plant nutrients, including deficiency of some nutrients or excessive levels of sodium $[17,18]$. One of the major imbalances is the alteration in calcium levels, which usually cause imbalance in the passive ion fluxes [19]. In addition, higher salt levels in plant tissues causes over generation of reactive oxygen species, which hinders certain metabolic functions of plants [20,21]. Productivity of such soils can be restored through rational management, which not only improves conditions for better crop establishment but also minimizes the global pressure for cultivatable land resources.

Different approaches have been employed hitherto to ameliorate saline soils including physical, chemical, biological, and engineering practices such as leaching with water, amending soils with chemicals, organic amendments (composts and farmyard manure), microbial amelioration, and phytoremediation [22-26]. The use of chemical amendments such as gypsum $\left(\mathrm{CaSO}_{4} \cdot 2 \mathrm{H}_{2} \mathrm{O}\right)$, calcium chloride $\left(\mathrm{CaCl}_{2}\right)$, calcite $\left(\mathrm{CaCO}_{3}\right)$, etc., have been found to be effective for amelioration of saline soil [26-28]. In addition, application of organic materials is a significant practice which can improve the productivity of saline soils through improved physical structure and microbial activities [29,30]. Addition of organic manure increases plant available nutrients while decreasing the concentrations of sodium, carbonates, and bicarbonates in soil solution, and hence promotes plant growth, making it a feasible amendment for amelioration of salty soils [26,31,32]. Reduced physical firmness of salt-affected soils is usually due to low organic matter content. Addition of organic materials, for example green manure, farmyard manure, poultry manure, compost, and food processing waste, can improve the physical firmness of soil [33]. However, organic sources contain somewhat low concentrations of readily available plant nutrients and are not instantly accessible to plants for consumption. Therefore, fortification of organic materials with essential plant nutrients, especially calcium to replace excessive sodium ions in saline soils, is of vital importance to restore productivity of salt-affected soils and to contribute in food security.

Canola (Brassica napus L.) is one of the world's major oilseed crops [34]. Canola is also moderately tolerant to salinity [35]; however, severe concentrations of soluble salts limits its production in arid and semi-arid regions. Therefore, it becomes imperative to sustain its production through wise management of saline soils. Application of organic amendments including animal manure is considered a traditional 
measure to rehabilitate such problematic soils [36,37]. However, the role of externally Ca-fortified animal manure (Ca-FCM) remains neglected, specifically how Ca-functionalized animal manure reverts the deleterious effects of salinity through improved growth, physiology, and nutrient homeostasis of canola, which is grown to provide the theoretical basis on the effects of applied amendments under varying levels of salinity. In the present study, Ca-fortified composted animal manure was evaluated to enhance growth, physiology, yield, nutrients, and redox homeostasis in canola under salt-affected soil conditions.

\section{Materials and Methods}

\subsection{Procurement of Calcium-Entrapped Composted Manure}

Animal (cow) manure was collected from the Directorate of Farms, UAF, Pakistan for compost production and was composted in a locally fabricated unit consisting of drier, crusher/grinder, and processor. The manure was air-dried for a couple of days to remove excess moisture and unwanted materials were sorted out manually. The organic material was transferred to a vessel (500 kg capacity)

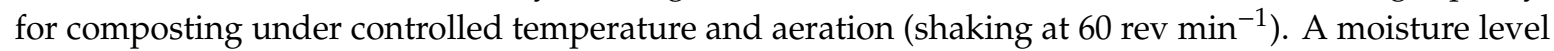
of $45-55 \%(v / w)$ of the organic material was maintained during the composting process. Urea and molasses as nitrogen and carbon sources were added at the rate of $1 \%(w / w)$ and $0.5(v / w)$ along with inoculum of a cellulase producing bacterial strain Bacillus sp. MN54 (10 $\left.\mathrm{CFU} \mathrm{mL}^{-1}\right)$ as decomposer. Temperature rose up to $65-70{ }^{\circ} \mathrm{C}$ in the composting unit during the second and third weeks of composting process and then reduced gradually to $30-35{ }^{\circ} \mathrm{C}$ after the fourth week of process. Then, Ca-based waste material of calcium ammonium nitrate (CAN) fertilizer was enriched at the rate of 1:1 ratio on dry weight basis $(w / w)$ to enhance the quality and nutritional value of the organic product. Co-composting was done for six weeks and organic material was analyzed on dry weight basis for carbon, total nitrogen, available phosphorus, and exchangeable potassium [38,39]. Calcium contents of co-composted organic product were determined through Atomic Absorption Spectrophotometer (Perkin Elmer Aanalyst-100, USA) having a graphite furnace equipped with Ca-cathode lamp. Basic physico-chemical features of test soil and Ca-FCM are given in Table 1.

Table 1. Basic physico-chemical properties of experimental soil and Ca-FCM used in the experiment.

\begin{tabular}{|c|c|c|c|}
\hline Soil Characteristics & Value & Ca-FCM Characteristics & Value \\
\hline Sand (\%) & $50 \pm 3.73$ & Carbon $\left(\mathrm{g} \mathrm{kg}^{-1}\right)$ & $108.7 \pm 7.4$ \\
\hline Silt (\%) & $35 \pm 2.21$ & Nitrogen $\left(\mathrm{g} \mathrm{kg}^{-1}\right)$ & $107.2 \pm 3.6$ \\
\hline Clay $(\%)$ & $15 \pm 2.15$ & Total P $\left(\mathrm{g} \mathrm{kg}^{-1}\right)$ & $166.0 \pm 2.2$ \\
\hline Textural class & Sandy clay loam & Olson $\mathrm{P}\left(\mathrm{mg} \mathrm{kg}^{-1}\right)$ & $133 \pm 8.7$ \\
\hline $\mathrm{pH}_{\mathrm{s}}$ & $7.33 \pm 0.17$ & $\mathrm{Ca}\left(\mathrm{g} \mathrm{kg}^{-1}\right)$ & $43.9 \pm 2.9$ \\
\hline $\mathrm{EC}_{\mathrm{e}}\left(\mathrm{dS} \mathrm{m}^{-1}\right)$ & $1.5 \pm 0.13$ & $\mathrm{pH}$ & $6.68 \pm 0.19$ \\
\hline Soluble $\mathrm{CO}_{3}{ }^{2-}\left(\mathrm{mmol}_{\mathrm{C}} \mathrm{L}^{-1}\right)$ & $0.8 \pm 0.1$ & $\begin{array}{l}\text { Cation exchange capacity } \\
\qquad\left(\mathrm{cmol}_{\mathrm{c}} \mathrm{kg}^{-1}\right)\end{array}$ & $51.20 \pm 2.1$ \\
\hline Soluble $\mathrm{HCO}_{3}{ }^{-}\left(\mathrm{mmol}_{\mathrm{C}} \mathrm{L}^{-1}\right)$ & 0 & & \\
\hline Organic matter $(\%)$ & $0.72 \pm 0.25$ & & \\
\hline Saturation percentage (\%) & $32 \pm 0.96$ & & \\
\hline Total nitrogen $(\%)$ & $0.046 \pm 0.01$ & & \\
\hline Extractable potassium $\left(\mathrm{mg} \mathrm{kg}^{-1}\right)$ & $112 \pm 4.13$ & & \\
\hline Available phosphorous (mg kg-1) & $3.45 \pm 1.10$ & & \\
\hline Cation exchange capacity $\left(\mathrm{cmol}_{\mathrm{C}} \mathrm{kg}^{-1}\right)$ & $9.12 \pm 2.1$ & & \\
\hline
\end{tabular}

Ca-FCM, calcium fortified composted animal manure. Values are mean of three repeats \pm SD.

All chemicals and reagents used were of analytical grade, provided by Sigma-Aldrich and Merck, Germany.

\subsection{Experimental Materials and Cultivation of Canola Crop}

A pot experiment was conducted at wire house, Institute of Soil and Environmental Sciences (ISES), University of Agriculture, Faisalabad (UAF), Pakistan. The soil for the experiment was collected from 
farm research area of Institute of Soil and Environmental Sciences (ISES), University of Agriculture, Faisalabad (UAF). After air drying, the soil was ground and passed through a 2-mm sieve and examined for different physical and chemical characteristics. Saline soil was artificially prepared by using sodium chloride $(\mathrm{NaCl})$ salt and by putting calculated values of soil EC (electrical conductivity) plus required EC in a quadratic equation [40]. Three EC levels were developed, i.e., original 1.5, 5, and $10 \mathrm{dS} \mathrm{m}^{-1}$, representing a total of 36 pots. Each pot was filled with $8 \mathrm{~kg}$ well sieved soil. Calcium-fortified composted animal manure (Ca-FCM) was applied at the rate of $1 \%, 2 \%$, and $3 \%(\mathrm{w} / \mathrm{w})$ besides untreated control. All the treatments were kept according to completely randomized design (CRD). Six seeds of canola (cv. Faisal) were raised in each pot and three plants per pot were maintained after germination. Seeds of canola cultivar were very kindly provided by Oil Seed Section, Ayub Agricultural Research Institute (AARI), Faisalabad, Pakistan. Recommended rates of (NPK) fertilizer were applied at the rate of 90:60:75 $\mathrm{kg} \mathrm{ha}^{-1}$ as urea, diammonium phosphate, and sulfate of potash, respectively [41].

\subsection{Physiological Attributes}

Physiological traits were measured after 45 days of sowing using infrared gas analyzer (IRGA) for photosynthetic rate, transpiration rate, stomatal conductance, and intrinsic $\mathrm{CO}_{2}$ concentration. For this purpose, two leaves (from top) of an individual plant from each pot were selected for physiological recordings. Relative chlorophyll measurement, in terms of SPAD value, was performed on third upper leaves with the help of chlorophyll meter (SPAD-502, Minolta, Japan) [42]. Each sample of leaf was dignified in at least six diverse spaces. Entirely stretched flag leaves (two leaves per plant and six leaves per pot) were used for measuring relative water contents and electrolyte leakage.

Relative water contents were calculated following the equation described by Teulat et al. [43]

$$
R W C(\%)=\frac{F W-D W}{F T W-D W} \times 100
$$

where FW is fresh weight, DW is oven-dried weight, and FTW is fully-turgid weight after dipping leaves in water for $24 \mathrm{~h}$ at $4{ }^{\circ} \mathrm{C}$.

Similarly, electrolyte leakage (EL) was measured using the formula designed by Lutts et al. [44];

$$
E L(\%)=\frac{E C 1}{E C 2} \times 100
$$

where EC1 is the electrical conductivity of solution in test tubes containing leaves and EC2 is electrical conductivity of solution in test tubes after autoclaving at $121^{\circ} \mathrm{C}$ for $20 \mathrm{~min}$.

\subsection{Harvesting and Agronomic Variables Measurements}

On the onset of maturity, agronomical parameters were recorded following standard methods. After harvesting the crop, shoot fresh weight was measured with the help of electrical balance and then kept in an oven (at $80^{\circ} \mathrm{C}$ for $48 \mathrm{~h}$ ) for drying. After oven drying, shoot dry weight was measured with the help of electrical balance. Later, the number of pods per plant was counted and the number of seeds per plant was estimated. Pods were separated from the shoots and net as well as 1000-grains weight were taken with the help of electrical balance. After crop removal, pots were flooded with water and kept for $24 \mathrm{~h}$. On the next day, soil was removed from the pots and roots were separated and then washed with tap water to record root characters. Root length was measured with the help of meter rod and root dry weight was measured by using electrical balance after oven drying at $65^{\circ} \mathrm{C}$.

\subsection{Measurement of Antioxidant Enzymes}

For the measurement of various enzymatic antioxidants, fresh leaf material was homogenized in an ice-cold solution comprising $0.2 \mathrm{M}$ potassium phosphate buffer $(\mathrm{pH} 7)$ having $0.1 \mathrm{mM}$ EDTA. Afterwards, six enzymatic antioxidants, namely ascorbate peroxidase (APX), catalase (CAT), glutathione peroxidase (GPX), glutathione reductase (GR), glutathione-S-transferase (GST), and superoxide 
dismutase (SOD), were measured following standard methods given by Nakano and Asada [45], Cakmak and Marschner [46], Smith et al. [47], Habig et al. [48], and Roth and Gilbert [49].

\subsection{Mineral Nutrition of Canola}

Various mineral nutrients of canola plants related to NPK and sodium were examined after digesting samples. For this purpose, shoot and leaf samples were air-dried and then oven-dried at $65^{\circ} \mathrm{C}$, grinded by grinder, and prepared for chemical analysis. Following the method given by Wolf [50], plant samples $(0.5 \mathrm{~g})$ were digested by adding $10 \mathrm{~mL}$ sulfuric acid and the flasks were kept overnight. The next day, $2 \mathrm{~mL}$ hydrogen peroxide was added and then retained on a hot plate for digestion maintaining temperature up to $300-350^{\circ} \mathrm{C}$, followed by dilution with distilled water and filtration using Whatman filter paper No. 42. Potassium $\left(\mathrm{K}^{+}\right)$and sodium $\left(\mathrm{Na}^{+}\right)$were measured directly from the digested solution by flame photometer [51], nitrogen (N) by Kjeldahl apparatus [52], and phosphorus (P) on spectrophotometer [53]. Afterwards, $\mathrm{Na}^{+} / \mathrm{K}^{+}$ratio in leaves of canola was determined.

\subsection{Statistical Analysis}

Data were analyzed through analysis of variance (ANOVA) using computer-based software statistics 8.1 (Statistix, Tallahassee, FL, USA) at $p \leq 0.05$. Standard deviation (SD) values were computed as an average of three replications of each treatment using "Microsoft excel 365 version". Treatment means were compared by using Tuckey's HSD test [54]. All graphs and correlation analyses were performed on Origin Pro version 9.1 computer-based software.

\section{Results}

\subsection{Growth and Yield Attributes}

Significant improvements in plant growth and yield attributes such as total biomass, shoot length, root dry weight, root length, straw weight, number of pods per plant, number of grains per pod, 1000-grains weight, and net grains weight were detected by the use of calcium-fortified composted animal manure (Ca-FCM) at three application levels, i.e., $1 \%, 2 \%$, and 3\%, under saline soil conditions relative to control. However, maximum increase in all parameters was recorded when Ca-FCM was applied at 3\% level. Applying 3\% Ca-FCM significantly increased total biomass of the plant up to $117 \%, 132 \%$, and $155 \%$ at EC $1.5,5$, and $10 \mathrm{dS} \mathrm{m}^{-1}$, respectively, relative to control (Table 2). In case of shoot length, 3\% Ca-FCM maximally increased the shoot length by $37 \%, 43 \%$, and $47 \%$ at EC 1.5, 5, and $10 \mathrm{dS} \mathrm{m}^{-1}$, respectively, relative to control.

Maximum increase up to $107 \%, 136 \%$, and $178 \%$ in root dry weight compared to control, was recorded through 3\% Ca-FCM application at EC 1.5, 5, and $10 \mathrm{dS} \mathrm{m}^{-1}$, respectively (Table 2). Similarly, 3\% Ca-FCM application increased root length up to $111 \%, 113 \%$, and $114 \%$ at EC levels 1.5, 5, and $10 \mathrm{dS} \mathrm{m}^{-1}$, respectively, relative to control.

Practicing 3\% Ca-FCM application significantly increased straw weight by $138 \%, 153 \%$, and $185 \%$ at EC 1.5, 5, and $10 \mathrm{dS} \mathrm{m}^{-1}$, respectively, over control. Likewise, maximum increase in number of pods per plant up to $109 \%, 132 \%$, and $159 \%$ was recorded by 3\% Ca-FCM application at EC levels 1.5, 5, and $10 \mathrm{dS} \mathrm{m}^{-1}$, respectively, over control (Table 2).

Likewise, amending soils with $3 \%$ Ca-FCM significantly increased number of grains per pod at EC levels $1.5,5$, and $10 \mathrm{dS} \mathrm{m}^{-1}$, which were $134 \%, 152 \%$, and $184 \%$, respectively, higher than un-amended control. The 1000-grains weight followed a similar trend where $3 \%$ Ca-FCM showed maximum increase in 1000-grains weight by $147 \%, 159 \%$, and $186 \%$ at EC $1.5,5$, and $10 \mathrm{dS} \mathrm{m}^{-1}$, respectively, compared to control. In the same way, $108 \%, 115 \%$, and $117 \%$ increase was recorded by application of $3 \%$ Ca-FCM at EC 1.5, 5, and $10 \mathrm{dS} \mathrm{m}^{-1}$, respectively, over control (Table 3). 
Table 2. The effect of calcium-fortified composted manure on growth parameters of canola under saline soils.

\begin{tabular}{|c|c|c|c|c|c|c|c|c|c|}
\hline \multirow{2}{*}{ Treatments } & \multicolumn{3}{|c|}{ Total Biomass (g) } & \multicolumn{3}{|c|}{ Shoot Length $(\mathrm{cm})$} & \multicolumn{3}{|c|}{ Root Dry Weight (g) } \\
\hline & $1.5 \mathrm{dS} \mathrm{m}^{-1}$ & $5 \mathrm{dS} \mathrm{m}^{-1}$ & $10 \mathrm{dS} \mathrm{m}^{-1}$ & $1.5 \mathrm{dS} \mathrm{m}^{-1}$ & $5 \mathrm{dS} \mathrm{m}^{-1}$ & $10 \mathrm{dS} \mathrm{m}^{-1}$ & $1.5 \mathrm{dS} \mathrm{m}^{-1}$ & $5 \mathrm{dS} \mathrm{m}^{-1}$ & $10 \mathrm{dS} \mathrm{m}^{-1}$ \\
\hline Control & $30.1 \pm 0.6 \mathrm{e}$ & $23.9 \pm 0.5 \mathrm{f}$ & $18.9 \pm 0.9 \mathrm{~g}$ & $101.8 \pm 1.5 \mathrm{e}$ & $86.4 \pm 0.6 \mathrm{~g}$ & $75.5 \pm 0.9 \mathrm{~h}$ & $6.7 \pm 0.2 \mathrm{e}$ & $4.7 \pm 0.2 \mathrm{~g}$ & $3.1 \pm 0.1 \mathrm{~g}$ \\
\hline $1 \% \mathrm{Ca}-\mathrm{FCM}$ & $46.3 \pm 1.9 c$ & $37.0 \pm 1.1 \mathrm{~d}$ & $30.0 \pm 0.8 \mathrm{e}$ & $115.5 \pm 1.6 \mathrm{c}$ & $101.5 \pm 0.5 \mathrm{e}$ & $91.1 \pm 0.6 \mathrm{f}$ & $9.9 \pm 0.3 c$ & $7.2 \pm 0.2 \mathrm{e}$ & $5.3 \pm 0.1 \mathrm{f}$ \\
\hline $2 \%$ Ca-FCM & $52.5 \pm 1.4 \mathrm{~b}$ & $45.8 \pm 1.9 \mathrm{c}$ & $39.5 \pm 1.5 \mathrm{~d}$ & $122.6 \pm 1.5 \mathrm{~b}$ & $113.95 \pm 0.7 \mathrm{~cd}$ & $98.8 \pm 2.5 \mathrm{e}$ & $11.3 \pm 0.6 b$ & $9.0 \pm 0.1 \mathrm{~d}$ & $6.8 \pm 0.4 \mathrm{e}$ \\
\hline \multirow[t]{2}{*}{ 3\% Ca-FCM } & $65.5 \pm 0.4 \mathrm{a}$ & $55.4 \pm 1.3 \mathrm{~b}$ & $48.0 \pm 0.7 \mathrm{c}$ & $139.9 \pm 0.8 \mathrm{a}$ & $123.3 \pm 0.6 \mathrm{~b}$ & $111.2 \pm 1.0 \mathrm{~d}$ & $13.9 \pm 0.4 \mathrm{a}$ & $11.0 \pm 0.1 \mathrm{~b}$ & $8.7 \pm 0.3 \mathrm{~d}$ \\
\hline & \multicolumn{3}{|c|}{ Root Length (cm) } & \multicolumn{3}{|c|}{ Straw Weight (g) } & \multicolumn{3}{|c|}{ No. of Pods (plant ${ }^{-1}$ ) } \\
\hline Control & $9.5 \pm 0.3 \mathrm{de}$ & $7.5 \pm 0.6 \mathrm{fg}$ & $6.1 \pm 0.5 \mathrm{~g}$ & $9.0 \pm 0.6 \mathrm{fg}$ & $7.7 \pm 0.3 \mathrm{gh}$ & $6.0 \pm 0.2 \mathrm{~h}$ & $115.3 \pm 1.5 \mathrm{~g}$ & $86.7 \pm 0.9 \mathrm{~h}$ & $64.6 \pm 0.3 \mathrm{i}$ \\
\hline $1 \%$ Ca-FCM & $13.1 \pm 0.5 c$ & $10.7 \pm 1.3 \mathrm{~d}$ & $8.2 \pm 0.9$ ef & $14.6 \pm 0.4 \mathrm{de}$ & $12.6 \pm 1.3 \mathrm{e}$ & $10.2 \pm 0.5 \mathrm{f}$ & $160.0 \pm 1.7 \mathrm{e}$ & $139.3 \pm 1.5 \mathrm{f}$ & $117.3 \pm 0.3 \mathrm{~g}$ \\
\hline $2 \%$ Ca-FCM & $15.1 \pm 0.6 b$ & $12.8 \pm 0.2 \mathrm{c}$ & $10.2 \pm 0.6 \mathrm{~d}$ & $17.0 \pm 0.8 c$ & $15.7 \pm 0.3 \mathrm{~cd}$ & $13.9 \pm 0.7 \mathrm{de}$ & $188.7 \pm 0.9 c$ & $160.0 \pm 1.2 \mathrm{e}$ & $138.7 \pm 0.9 \mathrm{f}$ \\
\hline 3\% Ca-FCM & $20.2 \pm 0.8 \mathrm{a}$ & $16.0 \pm 0.4 b$ & $13.1 \pm 0.3 c$ & $21.5 \pm 0.9 \mathrm{a}$ & $19.4 \pm 0.2 \mathrm{~b}$ & $17.2 \pm 1.1 \mathrm{c}$ & $241.0 \pm 0.6 \mathrm{a}$ & $200.7 \pm 0.7 b$ & $167.7 \pm 1.2 \mathrm{~d}$ \\
\hline
\end{tabular}

Quantities sharing similar letters are not significant with each other at $p \leq 0.05$. Data are mean of three replicates \pm SD. Ca-FCM, calcium-fortified composted animal manure.

Table 3. The effect of calcium-fortified composted manure on yield and physiological parameters of canola under saline soils.

\begin{tabular}{|c|c|c|c|c|c|c|c|c|c|}
\hline \multirow{2}{*}{ Treatments } & \multicolumn{3}{|c|}{ No. of Grains $\left(\operatorname{pod}^{-1}\right)$} & \multicolumn{3}{|c|}{1000 Grains Weight (g) } & \multicolumn{3}{|c|}{ Net Grains Weight (g) } \\
\hline & $1.5 \mathrm{dS} \mathrm{m}^{-1}$ & $5 \mathrm{dS} \mathrm{m}^{-1}$ & $10 \mathrm{dS} \mathrm{m}^{-1}$ & $1.5 \mathrm{dS} \mathrm{m}^{-1}$ & $5 \mathrm{dS} \mathrm{m}^{-1}$ & $10 \mathrm{dS} \mathrm{m}^{-1}$ & $1.5 \mathrm{dS} \mathrm{m}^{-1}$ & $5 \mathrm{dS} \mathrm{m}^{-1}$ & $10 \mathrm{dS} \mathrm{m}^{-1}$ \\
\hline Control & $10.7 \pm 0.3 \mathrm{e}$ & $8.3 \pm 0.9 \mathrm{f}$ & $6.3 \pm 0.7 \mathrm{f}$ & $2.2 \pm 0.1 \mathrm{efg}$ & $1.7 \pm 0.1 \mathrm{gh}$ & $1.2 \pm 0.1 \mathrm{~h}$ & $4.0 \pm 0.1 \mathrm{fgh}$ & $3.4 \pm 0.1 \mathrm{hi}$ & $2.9 \pm 0.1 \mathrm{i}$ \\
\hline $1 \% \mathrm{Ca}-\mathrm{FCM}$ & $16.0 \pm 0.6 \mathrm{c}$ & $13.3 \pm 1.2 \mathrm{~d}$ & $11.0 \pm 0.6 \mathrm{e}$ & $3.4 \pm 0.1 \mathrm{~cd}$ & $2.8 \pm 0.5 \mathrm{de}$ & $1.9 \pm 0.2 \mathrm{fg}$ & $5.3 \pm 0.2 \mathrm{de}$ & $4.6 \pm 0.1 \mathrm{efg}$ & $3.6 \pm 0.6 \mathrm{ghi}$ \\
\hline $2 \%$ Ca-FCM & $20.3 \pm 0.9 b$ & $16.3 \pm 0.7 \mathrm{c}$ & $13.7 \pm 0.3 \mathrm{~d}$ & $4.0 \pm 0.7 \mathrm{bc}$ & $3.3 \pm 0.1 \mathrm{~cd}$ & $2.5 \pm 0.1 \mathrm{ef}$ & $6.4 \pm 0.8 \mathrm{c}$ & $5.5 \pm 0.4 \mathrm{de}$ & $4.7 \pm 0.2 \mathrm{ef}$ \\
\hline \multirow[t]{2}{*}{$3 \% \mathrm{Ca}-\mathrm{FCM}$} & $25.0 \pm 0.6 \mathrm{a}$ & $21.0 \pm 0.6 \mathrm{~b}$ & $18.0 \pm 0.6 c$ & $5.4 \pm 0.1 \mathrm{a}$ & $4.4 \pm 0.1 \mathrm{~b}$ & $3.4 \pm 0.1 \mathrm{~cd}$ & $8.4 \pm 0.23 \mathrm{a}$ & $7.4 \pm 0.2 \mathrm{~b}$ & $6.2 \pm 0.3 \mathrm{~cd}$ \\
\hline & \multicolumn{3}{|c|}{ Relative Chlorophyll Contents (SPAD Value) } & \multicolumn{3}{|c|}{ Relative Water Contents (\%) } & \multicolumn{3}{|c|}{ Electrolyte Leakage (\%) } \\
\hline Control & $31.0 \pm 0.8 \mathrm{~g}$ & $24.5 \pm 0.8 \mathrm{~h}$ & $18.7 \pm 0.7 \mathrm{i}$ & $40.9 \pm 0.5 \mathrm{~h}$ & $32.4 \pm 0.7 \mathrm{i}$ & $25.4 \pm 0.9 \mathrm{j}$ & $40.0 \pm 1.2 \mathrm{c}$ & $48.1 \pm 1.1 b$ & $58.0 \pm 1.1 \mathrm{a}$ \\
\hline $1 \%$ Ca-FCM & $44.1 \pm 0.5 \mathrm{e}$ & $39.3 \pm 0.9 \mathrm{f}$ & $32.4 \pm 1.3 \mathrm{~g}$ & $54.7 \pm 0.5 \mathrm{f}$ & $49.1 \pm 0.6 \mathrm{~g}$ & $41.5 \pm 0.7 \mathrm{~h}$ & $28.4 \pm 0.7 \mathrm{e}$ & $35.6 \pm 0.6 \mathrm{~d}$ & $41.4 \pm 0.5 \mathrm{c}$ \\
\hline $2 \% \mathrm{Ca}-\mathrm{FCM}$ & $53.6 \pm 0.4 \mathrm{c}$ & $47.4 \pm 1.1 \mathrm{~d}$ & $42.8 \pm 2.7 \mathrm{e}$ & $66.9 \pm 0.9 c$ & $60.1 \pm 0.6 \mathrm{e}$ & $52.9 \pm 0.6 \mathrm{f}$ & $20.5 \pm 0.6 \mathrm{f}$ & $27.0 \pm 1.1 \mathrm{e}$ & $34.3 \pm 0.9 \mathrm{~d}$ \\
\hline $3 \%$ Ca-FCM & $65.8 \pm 0.8 \mathrm{a}$ & $57.7 \pm 0.4 b$ & $50.5 \pm 0.6 c$ & $85.7 \pm 0.9 a$ & $72.2 \pm 1.8 \mathrm{~b}$ & $63.1 \pm 0.6 \mathrm{~d}$ & $11.7 \pm 0.8 \mathrm{~h}$ & $16.6 \pm 0.5 \mathrm{~g}$ & $21.1 \pm 0.6 \mathrm{f}$ \\
\hline
\end{tabular}

Quantities sharing similar letters are not significant with each other at $p \leq 0.05$. Data are mean of three replicates \pm SD. Ca-FCM, calcium-fortified composted animal manure. 


\subsection{Physiological Parameters}

Data regarding physiological parameters indicate that application of Ca-FCM significantly increased photosynthetic rate, transpiration rate, stomatal conductance, intrinsic $\mathrm{CO}_{2}$ concentration, relative chlorophyll content (SPAD value), and RWC and decreased the EL at all EC levels compared to respective control. Maximum increase in photosynthetic rate by $102 \%, 127 \%$, and $156 \%$ was recorded through amendment of 3\% Ca-FCM at EC levels 1.5, 5, and $10 \mathrm{dS} \mathrm{m}^{-1}$, respectively, as compared to control (Figure 1A). Similar trends of the product (Ca-FCM) at different rates were observed in case of transpiration rate (Figure 1B). Maximum increase in stomatal conductance of $140 \%, 131 \%$, and $188 \%$ was recorded by applying $3 \%$ Ca-FCM under EC 1.5, 5, and $10 \mathrm{dS} \mathrm{m}^{-1}$, respectively, over control (Figure 1C). Likewise, application of 3\% Ca-FCM caused maximum increase in intrinsic $\mathrm{CO}_{2}$ concentration up to $43 \%, 41 \%$, and $51 \%$ at EC $1.5,5$, and $10 \mathrm{dS} \mathrm{m}^{-1}$, respectively, relative to un-amended control (Figure 1D). Maximum chlorophyll contents were recorded with 3\% Ca-FCM as $112 \%, 136 \%$, and $172 \%$ increase at EC $1.5,5$, and $10 \mathrm{dS} \mathrm{m}^{-1}$, respectively, over control was observed (Table 3). For RWC, maximum increase of $109 \%, 123 \%$, and 149\% were recorded by $3 \%$ Ca-FCM at EC $1.5,5$, and $10 \mathrm{dS} \mathrm{m}^{-1}$, respectively, relative to control (Table 3). Likewise, maximum decrease in EL was observed with the application of $3 \%$ Ca-FCM of $71 \%, 65 \%$, and $64 \%$ in EL at EC $1.5,5$, and $10 \mathrm{dS} \mathrm{m}^{-1}$, respectively, as compared to control (Table 3).
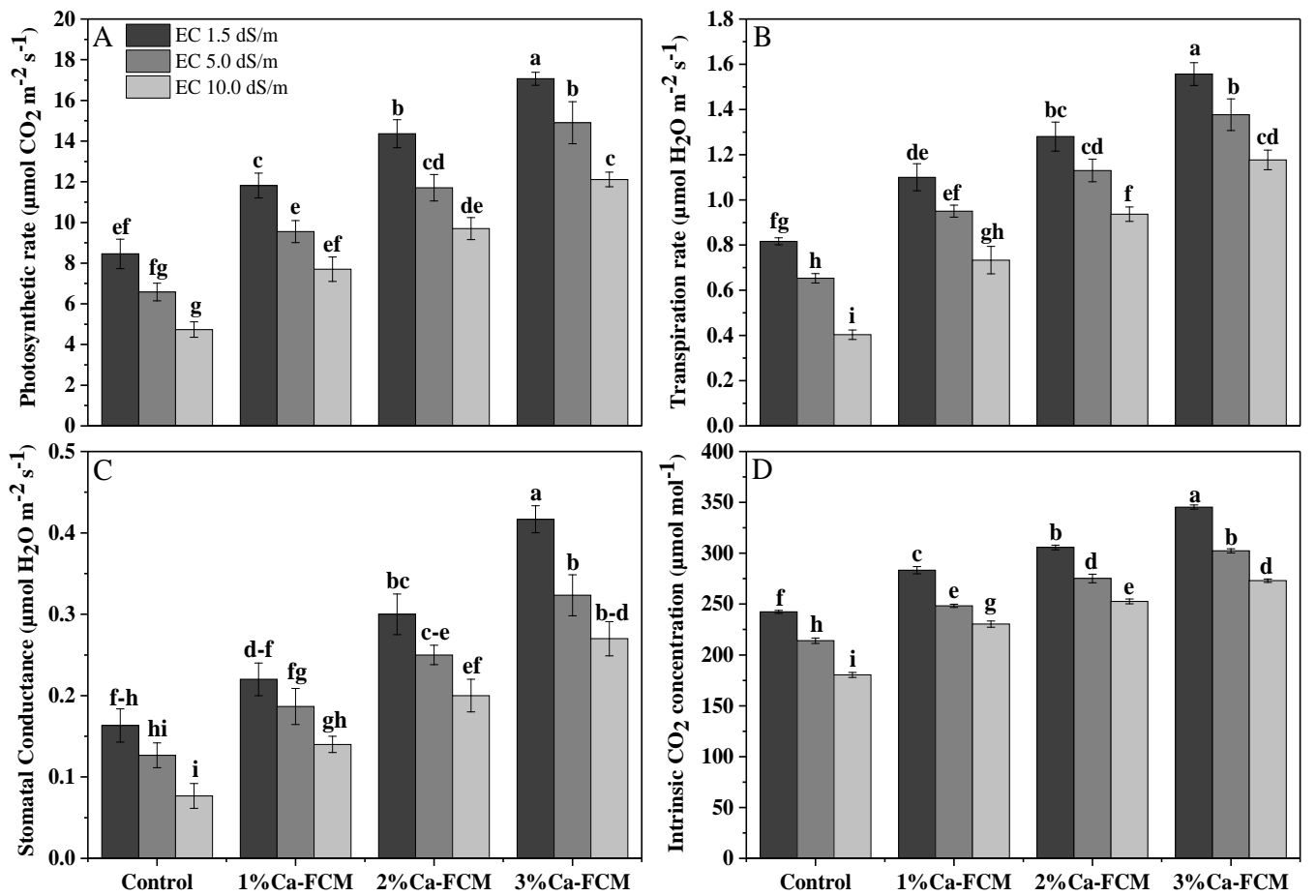

Figure 1. The effect of calcium-fortified composted manure on physiological parameters, photosynthetic rate (A), transpiration rate (B), stomatal conductance (C) and intrinsic $\mathrm{CO}_{2}$ concentration (D) of canola under saline soils. Quantities sharing similar letters are not significantly different from each other at $p \leq 0.05$. Data are mean of three replicates \pm SD. Ca-FCM, calcium-fortified composted animal manure.

\subsection{Enzymatic Antioxidant Attributes}

Data of antioxidant enzymes reveal that application of Ca-FCM product showed significant decrease in antioxidants attributes at all EC levels when compared with their respective controls. Amending soils with $3 \%$ Ca-FCM showed maximum decrease in ascorbate peroxidase (APX) activity of $71 \%, 62 \%$, and $58 \%$ at EC levels $1.5,5$, and $10 \mathrm{dS} \mathrm{m}^{-1}$, respectively, compared to control (Table 4). 
Table 4. The effect of calcium-fortified composted manure on antioxidant enzymes activity of canola under saline soils.

\begin{tabular}{|c|c|c|c|c|c|c|c|c|c|}
\hline \multirow{2}{*}{ Treatments } & \multicolumn{3}{|c|}{ Ascorbate Peroxidase (APX) } & \multicolumn{3}{|c|}{ Catalase (CAT) } & \multicolumn{3}{|c|}{ Glutathione Peroxidase (GPX) } \\
\hline & $1.5 \mathrm{dS} \mathrm{m}^{-1}$ & $5 \mathrm{dS} \mathrm{m}^{-1}$ & $10 \mathrm{dS} \mathrm{m}^{-1}$ & $1.5 \mathrm{dS} \mathrm{m}^{-1}$ & $5 \mathrm{dS} \mathrm{m}^{-1}$ & $10 \mathrm{dS} \mathrm{m}^{-1}$ & $1.5 \mathrm{dS} \mathrm{m}^{-1}$ & $5 \mathrm{dS} \mathrm{m}^{-1}$ & $10 \mathrm{dS} \mathrm{m}^{-1}$ \\
\hline Control & $42.2 \pm 1.0 \mathrm{c}$ & $51.5 \pm 1.3 b$ & $64.3 \pm 1.9 \mathrm{a}$ & $16.5 \pm 0.7 \mathrm{~cd}$ & $19.8 \pm 0.7 b$ & $24.2 \pm 0.7 \mathrm{a}$ & $58.8 \pm 1.7 \mathrm{c}$ & $75.0 \pm 1.5 \mathrm{~b}$ & $94.0 \pm 2.7 \mathrm{a}$ \\
\hline $1 \%$ Ca-FCM & $30.7 \pm 1.2 \mathrm{~d}$ & $40.5 \pm 1.1 \mathrm{c}$ & $50.9 \pm 1.3 \mathrm{~b}$ & $12.2 \pm 0.4$ ef & $15.8 \pm 0.6 \mathrm{~d}$ & $19.0 \pm 0.9 \mathrm{bc}$ & $43.0 \pm 1.2 \mathrm{~d}$ & $62.5 \pm 1.9 \mathrm{c}$ & $77.9 \pm 1.9 \mathrm{~b}$ \\
\hline $2 \%$ Ca-FCM & $22.6 \pm 1.1 \mathrm{ef}$ & $30.5 \pm 1.3 \mathrm{~d}$ & $39.6 \pm 1.5 c$ & $7.8 \pm 0.8 \mathrm{~g}$ & $11.9 \pm 0.3$ ef & $14.7 \pm 0.6 \mathrm{~d}$ & $32.3 \pm 1.5 \mathrm{e}$ & $47.8 \pm 1.7 \mathrm{~d}$ & $62.5 \pm 1.6 \mathrm{c}$ \\
\hline \multirow[t]{2}{*}{ 3\% Ca-FCM } & $12.1 \pm 0.9 \mathrm{~g}$ & $19.7 \pm 1.2 \mathrm{f}$ & $27.3 \pm 1.2 \mathrm{de}$ & $4.6 \pm 0.4 \mathrm{~h}$ & $7.5 \pm 0.3 \mathrm{~h}$ & $10.3 \pm 0.5 \mathrm{f}$ & $18.0 \pm 0.6 \mathrm{f}$ & $31.8 \pm 1.3 \mathrm{e}$ & $44.2 \pm 1.9 \mathrm{~d}$ \\
\hline & \multicolumn{3}{|c|}{ Glutathione Reductase (GR) } & \multicolumn{3}{|c|}{ Glutathione S-Transferase (GST) } & \multicolumn{3}{|c|}{ Superoxide Dismutase (SOD) } \\
\hline Control & $25.0 \pm 0.9 \mathrm{~cd}$ & $32.5 \pm 1.0 \mathrm{~b}$ & $38.8 \pm 1.4 \mathrm{a}$ & $11.1 \pm 0.4 \mathrm{de}$ & $13.9 \pm 0.4 b c$ & $17.8 \pm 0.5 \mathrm{a}$ & $175 \pm 6.6 \mathrm{c}$ & $217 \pm 7.1 \mathrm{~b}$ & $276 \pm 7.2 \mathrm{a}$ \\
\hline $1 \%$ Ca-FCM & $19.0 \pm 0.8 \mathrm{e}$ & $25.8 \pm 0.7 \mathrm{c}$ & $32.2 \pm 1.0 \mathrm{~b}$ & $8.6 \pm 0.3 \mathrm{f}$ & $11.7 \pm 0.4 \mathrm{~d}$ & $14.5 \pm 0.3 b$ & $134 \pm 8.3 \mathrm{de}$ & $175 \pm 9.9 c$ & $221 \pm 8.1 b$ \\
\hline $2 \%$ Ca-FCM & $13.3 \pm 0.7 \mathrm{f}$ & $20.0 \pm 0.6 \mathrm{e}$ & $26.6 \pm 0.9 c$ & $6.2 \pm 0.3 \mathrm{~g}$ & $8.9 \pm 0.3 \mathrm{f}$ & $12.2 \pm 0.4 \mathrm{~cd}$ & $97 \pm 5.2 \mathrm{f}$ & $137 \pm 4.1 \mathrm{~d}$ & $183 \pm 4.5 c$ \\
\hline 3\% Ca-FCM & $8.3 \pm 1.0 \mathrm{~g}$ & $14.0 \pm 1.1 \mathrm{f}$ & $20.2 \pm 1.0 \mathrm{de}$ & $3.8 \pm 0.3 \mathrm{~h}$ & $6.7 \pm 0.4 \mathrm{~g}$ & $9.4 \pm 0.4$ ef & $62 \pm 4.3 \mathrm{~g}$ & $103 \pm 7.6$ ef & $139 \pm 4.4 \mathrm{~d}$ \\
\hline
\end{tabular}

Quantities sharing similar letters are not significant with each other at $p \leq 0.05$. Data are mean of three replicates \pm SD. Ca-FCM, calcium-fortified composted animal manure. Antioxidant

enzymes activity was measured as nmol $\mathrm{min}^{-1} \mathrm{mg}^{-1}$ protein. 
Similarly, maximum decrease in catalase (CAT) activity of $72 \%, 62 \%$, and $57 \%$ was observed with 3\% Ca-FCM at EC 1.5, 5, and $10 \mathrm{dS} \mathrm{m}^{-1}$, respectively, relative to control (Table 4). In the case of glutathione peroxidase (GPX), 3\% Ca-FCM significantly decreased activity up to $69 \%, 58 \%$, and $53 \%$ at EC 1.5, 5, and $10 \mathrm{dS} \mathrm{m}^{-1}$, respectively, compared to control (Table 4). Applying 3\% Ca-FCM showed reduction in glutathione reductase (GR) activity by $67 \%, 57 \%$, and $48 \%$ at EC $1.5,5$, and $10 \mathrm{dS} \mathrm{m}^{-1}$, respectively, over control. Similar trend was observed in the case of glutathione $S$ transferase (GST) activity. Superoxide dismutase (SOD) activity was markedly decreased up to $65 \%, 53 \%$, and $50 \%$ by $3 \%$ Ca-FCM application at EC 1.5, 5, and $10 \mathrm{dS} \mathrm{m}^{-1}$, respectively, compared to control (Table 4).

\subsection{Chemical Parameters/Mineral Nutrition of Canola Plants}

A statistically significant increase in NPK concentrations was observed by $3 \%$ Ca-FCM treatment under all salinity levels (Figure 2A-C). The maximum $\mathrm{N}$ concentration in shoot was observed by $3 \%$ Ca-FCM of $105.82 \%, 152.69 \%$, and $172.92 \%$ at EC $1.5,5$, and $10 \mathrm{dS} \mathrm{m}^{-1}$, respectively, relative to control (Figure 2A). Similarly, 3\% Ca-FCM showed maximum increase in P concentration in shoot up to $122.60 \%, 155.04 \%$, and $168 \%$ at EC $1.5,5$, and $10 \mathrm{dS} \mathrm{m}^{-1}$, respectively, over control (Figure 2B). In the case of potassium concentration, 3\% Ca-FCM enhanced $\mathrm{K}$ in shoot by $114 \%, 129 \%$, and $177 \%$ at EC 1.5, 5, and $10 \mathrm{dS} \mathrm{m}^{-1}$, respectively, compared to control (Figure 2C). Conversely, it was found that maximum decrease in $\mathrm{Na}^{+} / \mathrm{K}^{+}$ratio of leaves was observed with $3 \%$ Ca-FCM of $83 \%, 78 \%$, and $71 \%$ at EC 1.5, 5, and $10 \mathrm{dS} \mathrm{m}^{-1}$, respectively, compared to un-amended control. Application of $1 \%$ Ca-FCM revealed a minor decrease in $\mathrm{Na}^{+} / \mathrm{K}^{+}$ratio of leaves under saline soil conditions (Figure 2D). Interestingly, we found significant positive correlations among $\mathrm{Na}^{+} / \mathrm{K}^{+}$ratio of leaves and plant growth (Figure $3 \mathrm{~A}$ ), physiological parameters (Figure 3B), plant nutritional parameters (Figure 3C), and antioxidant defense system (Figure 3D) of canola under the salinity stress.
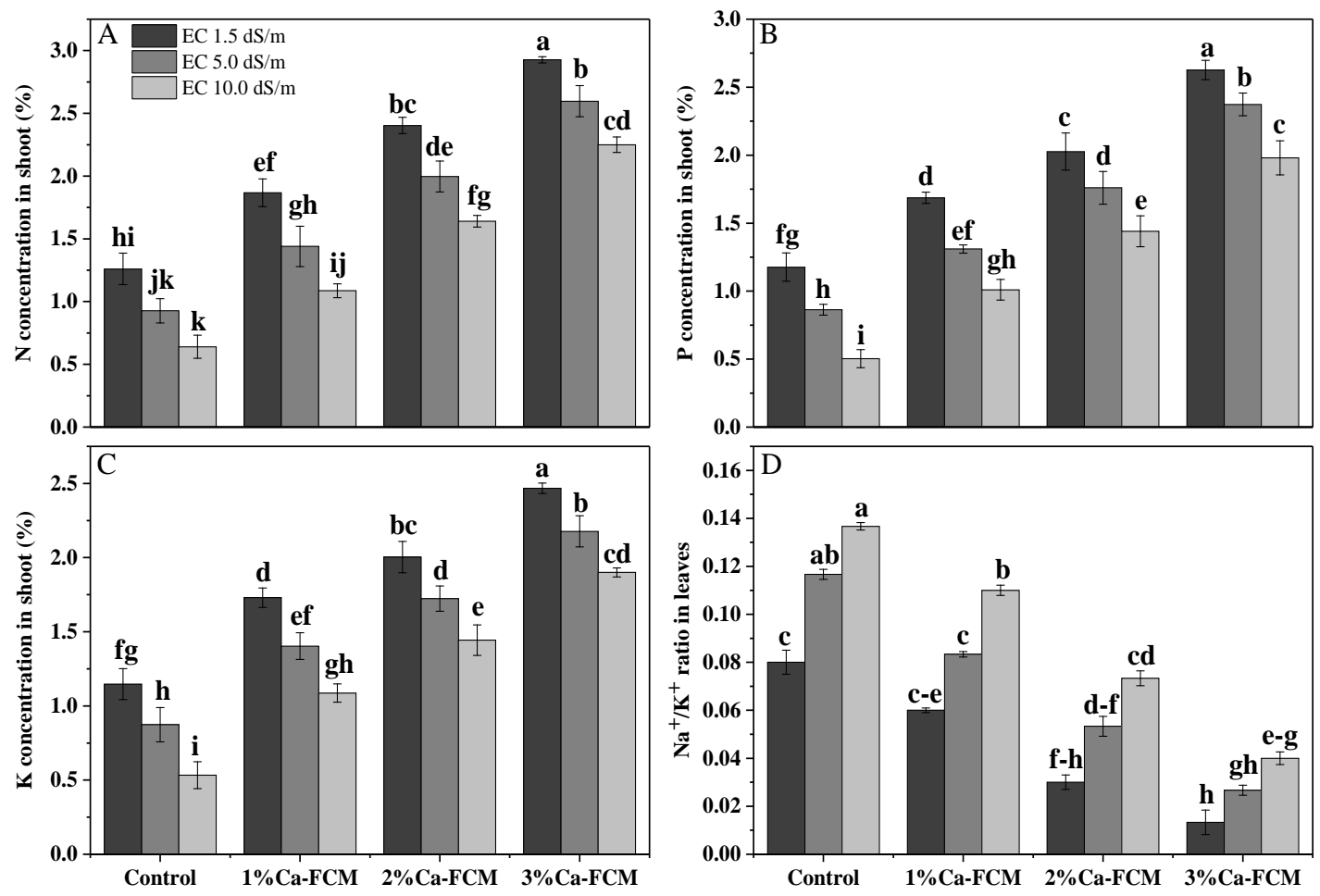

Figure 2. The effect of calcium-fortified composted manure on nutritional parameters, $\mathrm{N}$ concentration (A), P concentration (B), $\mathrm{K}$ concentration (C) and $\mathrm{Na}^{+} / \mathrm{K}^{+}$ratio in leaves (D) of canola under saline soil. Quantities sharing similar letters are not significantly different from each other at $p \leq 0.05$. Data are mean of three replicates \pm SD. Ca-FCM, calcium-fortified composted animal manure. 

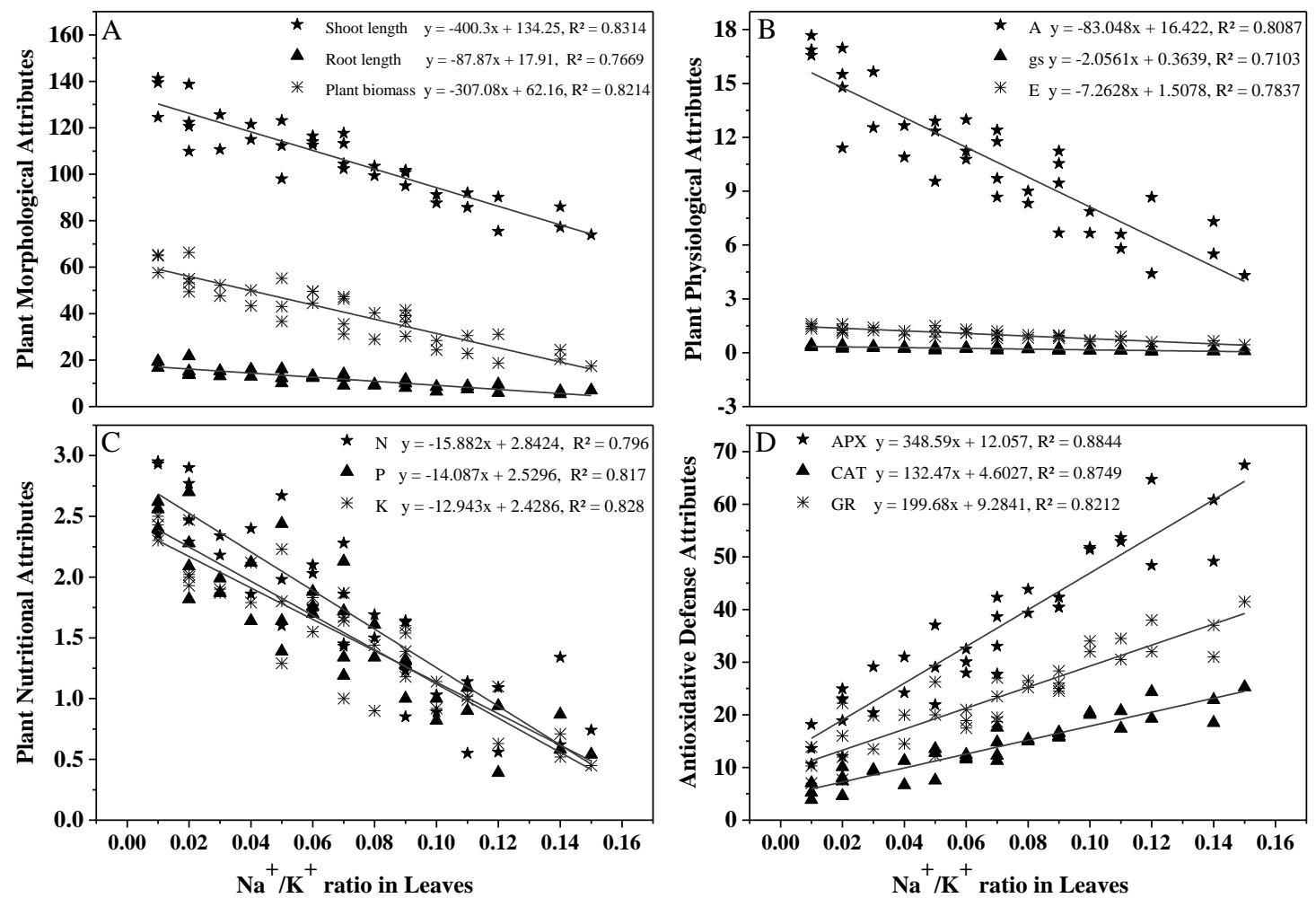

Figure 3. Regression relationship among $\mathrm{Na}^{+} / \mathrm{K}^{+}$ratio in leaves and plant morphological (A), physiological (B), nutritional parameters (C), and antioxidant defense attributes (D) of canola under saline soil conditions. A, photosynthetic rate; E, transpiration rate; gs, stomatal conductance.

\section{Discussion}

Soil salinity is one of the key classical issues that retards the growth of plants and metabolic rate, instigating severe loss of production and requiring wise management for sustainable crop production [55-57]. Soil salinization is one of the most important factors that contribute to degradation of land and decline in yield of crops [58]. The negative possessions of salinization are strengthened by inadequate soil organic matter levels $[59,60]$. For amelioration of saline soils, replacement of ions responsible for salinity, either chemically or by addition of organic materials, is found to be worthwhile [61]. Various organic amendments such as animal manure, crop residues, and sewage sludge are being used as sources of nutrients for improving the quality of saline soils $[60,62]$.

Salinity disturbs the crop growth by specific ion toxicity, osmotic stress, and nutrient imbalances $[14,63]$. The results of the present study showed that salts stress significantly decreased all the growth and yield parameters of canola. These results are in agreement with Shahzad et al. [64], who reported decreased growth attributes of maize grown under varying salinity levels. Application of organic amendments have been advocated for its known positive effects in salinity amelioration. We found that application of Ca-FCM significantly improved all the agronomical attributes of canola under different salinity levels. More precisely, observed agronomical attributes such as plant biomass, shoot length, root dry weight, root length, straw weight, number of pods per plant, number of grains per pod, 1000-grains weight, and net grains weight were significantly enhanced by application of 3\% Ca-FCM at EC 1.5, 5, and $10 \mathrm{dS} \mathrm{m}^{-1}$ as compared to its respective controls. Previously, substantial literature confirmed the optimistic relationship of organic material and increased growth and yield of maize, sunflower, wheat, rice, and holy basil (an aromatic crop) [26,65-68]. Increased growth and grain yield in the present experiment might be attributable to improved availability of mineral nutrients to plants due to applied composted manure [69]. It has been established that varying levels and kinds of salts present affect the fresh and dry biomass of plants [64,70]. Salt stress considerably 
decreased the biomass of canola but addition of organic matter through animal manure might have improved physico-chemical properties, which as a result might have improved physiology of plant and eventually growth and yield parameters examined during this study [26,31]. One possible explanation might be due to the role of organic sources such as composted animal manure in increasing biological activities of soil, which are linked to cycling of nutrients and eventually enhanced growth and yield parameters [71,72]. Moreover, composted manure application in the current study might have released organic acids that dissolved some of the $\mathrm{CaCO}_{3}$ present in soil and replaced $\mathrm{Na}^{+}$by $\mathrm{Ca}^{2+}$.

Salinity stress causes reduction in physiological parameters and hence the photosynthetic machinery of the plants. In the present study, salinity stress reduced physiological parameters such as photosynthetic rate, stomatal conductance, intrinsic $\mathrm{CO}_{2}$ concentration, transpiration rate, relative chlorophyll contents, and relative water contents. However, application of Ca-FCM, particularly at the rate of 3\%, significantly increased physiological attributes under normal as well as saline soil conditions. A significant decrease in electrolyte leakage was observed with the application of $3 \%$ $\mathrm{Ca}-\mathrm{FCM}$ as compared to respective control. The influence on photosynthesis can be assessed from the effect on photosynthetic pigments. It has been described in certain studies that salinity stress causes decline in photosynthetic pigments of plants $[26,64,73]$. The decrease in gaseous exchange attributes in the current study might be associated with increased salinity-induced osmotic stress that rendered the growing plants out of water and hampered the rate of transpiration, which further excavated water and $\mathrm{CO}_{2}$ supply for normal photosynthesis [74]. This might also be due to osmotic and hormonal imbalances created by the generation of reactive oxygen species (ROS) in plant cells that impaired carbohydrate metabolism and hence the photosynthetic efficiency $[75,76]$. However, in the present study, application of Ca-FCM was found to be an effective amendment for enhancing photosynthetic rate of plants under saline soil conditions. Several other studies have confirmed the adverse effects of salinity on water relations of growing plants [77,78]. We found decreased values of relative water contents in plants grown under increasing order of salt stress, but the applied amendments improved relative water contents. Recently, Niamat et al. [26] observed improved water relations of maize grown in varying salinity levels in terms of decreased electrolyte leakage and increased relative water contents under the application of calcium-enriched compost (Ca-FC).

Increased antioxidant activities under stress conditions are imperative for plants to cope with the oxidative stress caused by higher ROS levels [79]. The current findings reveal higher levels of antioxidant activities with increasing levels of salinity stress. Previously, it was stated that enhanced antioxidant activities help plants manage oxidative stress caused by ROS [80]. These findings are further substantiated with the findings of Ramzani et al. [81-85]. However, in the present study, the activities of antioxidants were decreased due to the application of Ca-FCM, especially at the rate of $3 \%$. These results are in agreement with the findings of various researchers [86-88]. The antioxidant defense responses were improved in Chenopodium quinoa through application of acidified compost under salinity stress [81] that might have scavenged ROS generation and ultimately enhanced plants resistance against salinity.

While it is well established that higher levels of $\mathrm{Na}^{+}$ions hinder uptake of other essential key nutrients by plants [89], the current findings reveal that the application of Ca-FCM prominently increased the concentrations of essential plant nutrients. This agrees with previous studies that organic amendments enhance the availability of essential nutrients [90,91]. In the present study, especially the application of 3\% Ca-FCM significantly enhanced nitrogen, phosphorus, and potassium concentrations in shoot under normal as well as saline soil conditions. These results are substantiated with Erdal et al. [92], who described that accumulation of nutrients in plants were boosted by using mixture of chemical and organic fertilizers. In addition, nitrogen and phosphorus concentrations in plants were improved with the application of rock phosphate mixed with organic fertilizers $[93,94]$. In the current study, maximum $\mathrm{Na}^{+}$concentration was observed in control (no-amendment) and minimum concentration was found in Ca-FCM at the rate of 3\% at all salinity levels. These results are in line with Shahzad et al. [64], who found increasing $\mathrm{Na}^{+}$concentrations in plant tissues with the increase 
in salinity levels. The increased $\mathrm{Na}^{+}$concentration in different plant parts (root, shoot, and grains) in control plants might be associated with the increased $\mathrm{Na}^{+}$ions in soil and rooting medium [95]. Moreover, maximum $\mathrm{K}^{+}$was found in plants treated with Ca-FCM in normal as well as in salt-stressed soil. This might be due to the applied composted manure that increased the cation exchange capacity (CEC) of the soils, which in turn limited the entry of $\mathrm{Na}^{+}$on exchange sites leading to increased $\mathrm{K}^{+}$for plant uptake [33,71].

Organic amendments differ in their effects on concentration of soluble cations. The application of organic amendments would most likely increase the amount of calcium $\left(\mathrm{Ca}^{2+}\right)$, derived from calcium carbonate $\left(\mathrm{CaCO}_{3}\right)$, due to organic acids formation [96]. Moreover, potassium $\left(\mathrm{K}^{+}\right)$and sodium $\left(\mathrm{Na}^{+}\right)$ions on soil colloids might be exchanged by $\mathrm{Ca}^{2+}$ ions and leached from the soil. The current study showed that the application of Ca-FCM significantly decreased $\mathrm{Na}^{+}$concentration in leaves and increased $\mathrm{K}^{+}$concentration in shoot under saline conditions. These results are in line with Li-ping et al. [37], who found decreased $\mathrm{Na}^{+}$-mediated stress to plants under applied cow dung and other ameliorants. It has previously been described that $\mathrm{Ca}^{2+}$ could modify the structure of soil by developing cationic channels between clay particles and organic material $[97,98]$. In addition, calcium ions can impede the scattering of clay and distraction of aggregates via interchanging sodium and magnesium ions in clay microsites and aggregates [99]. Recently, Huang et al. [98] indicated that application of organic fertilization could enhance the availability of $\mathrm{Ca}$ for $\mathrm{C}$ binding, possibly by forming organo-Ca complexes, which in turn improve soil aggregation and stabilization of soil organic carbon pool consequently improved plant growth, yield, and nutrients homeostasis, as revealed in the present study through application of Ca-FCM. In the current study, $\mathrm{Na}^{+} / \mathrm{K}^{+}$ratio in leaves was considerably decreased under the applied Ca-FCM at 3\% level in original and saline soils, which might be related to restricted uptake of $\mathrm{Na}^{+}$ions into plant. Previously, it was recommended that organic amendments such as farmyard manure be used as chelating agents for refining toxic salts, especially sodium $\left(\mathrm{Na}^{+}\right)$and chloride $\left(\mathrm{Cl}^{-}\right)$ions $[27,33]$.

\section{Conclusions}

This paper summarizes that application of 3\% Ca-FCM significantly improved the growth, yield, physiological, and nutritional parameters of canola grown under varying levels of salt stress. Conversely, application of 3\% Ca-FCM significantly reduced the $\mathrm{Na}^{+} / \mathrm{K}^{+}$ratio in canola leaves under saline soil conditions, indicating amelioration of salt-induced growth, physiological and nutritional disturbances of canola crop.

Author Contributions: Conceptualization, M.N., A.M., and J.-T.C.; methodology, M.N., M.Y., H.S., and M.R.; software, M.K. and A.M.; validation, M.N., M.Y., and Z.A.; formal analysis, A.M., M.K., and B.N.; investigation, H.S. and A.M.; resources, M.N. and J.-T.C.; data curation, H.S., S.A., and B.N.; writing-original draft preparation, M.N. and H.S.; writing-review and editing, A.M., M.Y., Z.Z., M.K., and S.A.; and supervision, M.N. and Z.A. All authors have read and agreed to the published version of the manuscript.

Funding: The present study received no external funding.

Acknowledgments: The authors are highly thankful to Ayub Agricultural Research Institute for provision of canola seeds. Furthermore, we are grateful to Directorate of Farms, UAF, Pakistan for provision of animal manure.

Conflicts of Interest: The authors declare that there is no conflict of interest among all authors either financially or otherwise.

\section{References}

1. Ashraf, M.; Athar, H.R.; Harris, P.J.C.; Kwon, T.R. Some prospective strategies for improving crop salt tolerance. Adv. Agron. 2008, 97, 45-110.

2. Ali, M.A.; Naveed, M.; Mustafa, A.; Abbas, A. The good, the bad, and the ugly of rhizosphere microbiome. In Probiotics and Plant Health; Springer: Singapore, 2017; pp. 253-290.

3. Izadi, M.H.; Rabbani, J.; Emam, Y.; Pessarakli, M.; Tahmasebi, A. Effects of salinity stress on physiological performance of various wheat and barley cultivars. J. Plant Nutr. 2014, 37, 520-531. [CrossRef] 
4. Hmaeid, N.; Wali, M.; Mahmoud, O.M.; Pueyo, J.J.; Ghnaya, T.; Abdelly, C. Efficient rhizobacteria promote growth and alleviate $\mathrm{NaCl}$-induced stress in the plant species Sulla carnosa. Appl. Soil Ecol. 2018. [CrossRef]

5. Summer, M.E. Handbook of Soil Science; CRC Press: Boca Raton, FL, USA, 2000.

6. Flowers, T.J.; Yeo, A.R. Breeding for salinity resistance in crop plants. Aust. J. Plant Phy. 1995, $22,875-884$. [CrossRef]

7. Lakhdar, A.; Rabhi, M.; Ghnaya, T.; Montemurro, F.; Jedidi, N.; Abdelly, C. Effectiveness of compost use in salt-affected soils. J. Hazar. Mater. 2009, 171, 29-37. [CrossRef]

8. Supper, S. Verstecktes Wasser. Sustainable Austria-Nachhaltiges Österreich, Nr. 2003. 25 December 2003. Available online: file://C:/Users/mnaveed/Desktop/Dr.\%20Shaukat\%20ALP/Haroon\%20Paper/SusA25.pdf (accessed on 15 December 2019).

9. Athar, H.R.; Ashraf, M. Strategies for crop improvement against salinity and drought stress: An overview. In Salinity and Water Stress; Springer: Dordrecht, The Netherlands, 2009; pp. 1-16.

10. Akram, M.; Ashraf, M.Y.; Ahmad, R.; Waraich, E.A.; Iqbal, J.; Mohsan, M. Screening for salt tolerance in maize (Zea mays L.) hybrids at an early seedling stage. Pak. J. Bot. 2010, 42, 141-154.

11. Lodhi, A.; Arshad, M.; Azam, F.; Sajjad, M.H. Changes in mineral and mineralizable N of soil incubated at varying salinity, moisture and temperature regimes. Pak. J. Bot. 2009, 41, 967-980.

12. Grewal, H.S.; Norrish, S.; Cornish, P. Subsoil salts affect root function, shoot growth and ionic balance of wheat plants. New directions for a diverse planet. In Proceedings of the 4 th International Crop Science Congress, Brisbane, Australia, 26 September-1 October 2004.

13. Aliu, S.; Rusinovci, I.; Fetahu, S.; Gashi, B.; Simeonovska, E.; Rozman, L. The effect of salt stress on the germination of maize (Zea mays L.) seeds and photosynthetic pigments. Acta Agric. Slov. 2015, 105, 85-94. [CrossRef]

14. Gong, D.H.; Wang, G.Z.; Si, W.T.; Zhou, Y.; Liu, Z.; Jia, J. Effects of salt stress on photosynthetic pigments and activity of ribulose- 1,5-bisphosphate carboxylase/oxygenase in Kalidium foliatum. Russian J. Plant Physiol. 2018, 65, 98-103. [CrossRef]

15. Reitz, D.N.; Haynes, R.J. Effect of irrigation induced salinity and sodicity on soil microbial activity. Soil Biol. Biochem. 2003, 35, 845-854. [CrossRef]

16. Vimal, S.R.; Singh, J.S.; Arora, N.K.; Singh, S. Soil-plant-microbe interactions in stressed agriculture management: A review. Pedosphere 2017, 27, 177-192. [CrossRef]

17. Kaya, C.; Kirnak, H.; Higgs, D.; Saltali, K. Supplementary calcium enhances plant growth and fruit yield in strawberry cultivars grown at high $(\mathrm{NaCl})$ salinity. Sci. Hortic. 2002, 93, 65-74. [CrossRef]

18. Eraslan, F.; Inal, A.; Savasturk, O.; Gunes, A. Changes in antioxidative system and membrane damage of lettuce in response to salinity and boron toxicity. Sci. Hort. 2007, 114, 5-10. [CrossRef]

19. Gao, Y.; Zhang, G. A calcium sensor calcineurin B-like 9 negatively regulates cold tolerance via calcium signaling in Arabidopsis thaliana. Plant Signal Behav. 2019, 14, e1573099. [CrossRef] [PubMed]

20. Shrivastava, P.; Kumar, R. Soil salinity: A serious environmental issue and plant growth promoting bacteria as one of the tools for its alleviation. Saudi J. Biol. Sci. 2015, 22, 123-131. [CrossRef]

21. Yang, Z.; Wang, C.; Xue, Y.; Liu, X.; Chen, S.; Song, C.; Yang, Y.; Guo, Y. Calcium-activated 14-3-3 proteins as a molecular switch in salt stress tolerance. Nat. Commun. 2019, 10, 1199. [CrossRef]

22. Zhu, Q.; Zhang, M.; Ma, Q. Copper-based foliar fertilizer and controlled release urea improved soil chemical properties: Plant growth and yield of tomato. Sci. Hort. 2012, 143, 109-114. [CrossRef]

23. Long, X.H.; Zhao, J.; Liu, Z.P.; Rengel, Z.; Liu, L.; Shao, H.B.; Tao, Y.A. Applying geostatistics to determine the soil quality improvement by Jerusalem artichoke in coastal saline zone. Ecol. Eng. 2014, 70, 319-326. [CrossRef]

24. Shalaby, O.A.E.S. Alleviation of salinity stress in red cabbage plants by urea and sulfur applications. J. Plant Nutr. 2018, 41, 1597-1603. [CrossRef]

25. Ansari, F.A.; Ahmad, I.; Pichtel, J. Growth stimulation and alleviation of salinity stress to wheat by the biofilm forming Bacillus pumilus strain FAB10. Appl. Soil Ecol. 2019, 143, 45-54. [CrossRef]

26. Niamat, B.; Naveed, M.; Ahmad, Z.; Yaseen, M.; Ditta, A.; Mustafa, A.; Rafique, M.; Bibi, R.; Sun, N.; Xu, M. Calcium-Enriched Animal Manure Alleviates the Adverse Effects of Salt Stress on Growth, Physiology and Nutrients Homeostasis of Zea mays L. Plants 2019, 8, 480. [CrossRef] [PubMed]

27. Hanay, A.; Büyüksönmez, F.; Kızıloglu, F.M.; Canbolat, M.Y. Reclamation of saline-sodic soils with gypsum and MSW compost. Compos. Sci. Utili. 2004, 12, 175-179. [CrossRef] 
28. Wang, J.M.; Yang, P.L.; Zhang, J.G.; Shi, Y. Salinity effect on sunflower at seedling stage during improving sodic soil reclaimed with by-product from flue gas desulphurization (BFGD). Trans. CSAE 2005, 21, 33-37. (In Chinese)

29. Wachendorf, C.; Taube, F.; Wachendorf, M. Nitrogen leaching from $15 \mathrm{~N}$ labelled cow urine and dung applied to grassland on a sandy soil. Nutr. Cycl. Agroecosyst. 2005, 73, 89-100. [CrossRef]

30. Melero, S.; Madejon, E.; Herencia1, J.F.; Ruiz, J.C. Biochemical properties of two different textured soils (loam and clay) after the addition of two different composts during conversion to organic farming. Span. J. Agric. Resea. 2007, 5, 593-604. [CrossRef]

31. Njoku, K.L.; Akinola, M.O.; Oboh, B.O. Growth and performance of glycine max L. (Merrill) grown in crude oil contaminated soil augmented with cow dung. Life Sci. J. 2008, 5, 89-93.

32. Rajendran, K.; Tester, M.; Roy, S.J. Quantifying the three main components of salinity tolerance in cereals. Plant Cell Environ. 2009, 32, 237-249. [CrossRef]

33. Tejada, M.; Garcia, C.; Gonzalez, J.L.; Hernandez, M.T. Use of organic amendment as a strategy for saline soil remediation: Influence on the physical, chemical and biological properties of soil. Soil Biol. Biochem. 2006, 38, 1413-1421. [CrossRef]

34. Iniguez-Luy, F.L.; Federico, M.L. The genetics of Brassica napus L. In Genetics and Genomics of the Brassicaceae; Bancroft, I., Schmidt, R., Eds.; Springer: Dordrecht, The Netherlands; New York, NY, USA; Heidelberg, Germany; London, UK, 2011; pp. 291-322.

35. Ashraf, M.; McNeilly, T. Salinity tolerance in Brassica oilseeds. Crit. Rev. Plant Sci. 2004, 23, $157-174$. [CrossRef]

36. Supparattanapan, S.; Saenjan, P.; Quantin, C.; Maeght, J.L.; Grunberger, O. Salinity and organic amendment effects on methane emission from a rain-fed saline paddy field. Soil Sci. Plant Nutr. 2009, 55, 142-149. [CrossRef]

37. Liu, L.-P.; Long, X.-H.; Shao, H.-B.; Liu, Z.-P.; Tao, Y.; Suo, Z.Q.; Zong, J.-Q. Ameliorants improve saline-alkaline soils on a large scale in northern Jiangsu Province, China. Ecol. Eng. 2015, 1, 328-334.

38. Nelson, D.W.; Sommers, L.E. Total carbon, Organic carbon and Organic matter. In Methods of Soil Analysis; Part 3; Book Series 5; Sparks, D.L., Page, A.L., Helmke, P.A., Loeppert, R.H., Soltanpour, P.N., Tabatabai, M.A., Johnston, C.T., Sumner, M.E., Eds.; SSSA: Madison, WI, USA, 1996; pp. 961-1010.

39. Estefan, G.; Sommer, R.; Ryan, J. Methods of Soil, Plant and Water Analysis: A Manual for the West Asia and North Africa Region, 3rd ed.; ICARDA: Beirut, Lebanon, 2013.

40. Richards, L.A. Diagnosis and improvement of saline and alkali soils. In Agriculture Handbook; USDA: Washington, DC, USA, 1954; p. 60.

41. Saeed, Z.; Naveed, M.; Imran, M.; Bashir, M.A.; Sattar, A.; Mustafa, A. Combined use of Enterobacter sp. MN17 and zeolite reverts the adverse effects of cadmium on growth, physiology and antioxidant activity of Brassica napus. PLoS ONE 2019, 14, e0213016. [CrossRef] [PubMed]

42. Ehsanzadeh, P.; Nekoonam, M.S.; Azhar, J.N.; Pourhadian, H.; Shaydaee, S. Growth, chlorophyll and cation concentration of tetraploid wheat on a solution high in sodium chloride salt: Hulled versus free-threshing genotypes. J. Plant Nutr. 2009, 32, 58-70. [CrossRef]

43. Teulat, B.; Zoumarou-Wallis, N.; Rotter, B.; Ben Saleem, M.; Bahri, H.; This, D. QTL for relative water content in field-grown barley and their stability across Mediterranean environments. Theorati. Appl. Genet. 2003, 108, 181-188. [CrossRef] [PubMed]

44. Lutts, S.; Guerrier, G. Peroxidase activities of two rice cultivars differing in salinity tolerance as affected by proline and $\mathrm{NaCl}$. Biol. Plant. 1995, 37, 577. [CrossRef]

45. Nakano, Y.; Asada, K. Hydrogen peroxide is scavenged by ascorbate-specific peroxidase in spinach chloroplasts. Plant Cell Physiol. 1981, 22, 867-880.

46. Cakmak, I.; Marschner, H. Magnesium deficiency and high light intensity enhance activities of superoxide dismutase, ascorbate peroxidase, and glutathione reductase in bean leaves. Plant Physiol. 1992, 98, 1222-1227. [CrossRef]

47. Smith, I.K.; Vierheller, T.L.; Thorne, C.A. Assay of glutathione reductase in crude tissue homogenates using 5,5'-dithiobis (2-nitrobenzoic acid). Anal. Biochem. 1988, 175, 408-413. [CrossRef]

48. Habig, W.H.; Pabst, M.J.; Jakoby, W.B. Glutathione-S-transferase, the first enzymatic step in mercapturic acid formation. J. Biol. Chem. 1974, 249, 7130-7139. 
49. Roth, E.F.J.; Gilbert, H.S. Pyrogallol assay for SOD: Absence of a glutathione artifact. Anal. Biochem. 1984, 137, 50-53. [CrossRef]

50. Wolf, B. A comprehensive system of leaf analyses and its use for diagnosing crop nutrient status. Commun. Soil Sci. Plant Anal. 1982, 13, 1035-1059. [CrossRef]

51. Chapman, H.D.; Pratt, P.F. Method of Analysis for Soil, Plants and Waters; University of California Division Agriculture Science: Berkeley, CA, USA, 1961.

52. Jackson, M.L. Chemical composition of soil. In Chemistry of Soil; Bean, F.E., Ed.; Van Nostrand Reinheld Co.: New York, NY, USA, 1962; pp. 71-144.

53. Olsen, S.R.; Cole, C.V.; Watanabe, F.S.; Dean, L.A. Estimation of Available Phosphorus in Soils by Extraction with Sodium Bicarbonate; Circular No. 939, USDA; US Government Printing Office: Washington, DC, USA, 1954.

54. Steel, R.G.D.; Torrie, J.H.; Dickey, D.A. Principles and Procedures of Statistics. A Bio Metrical Approach; McGraw Hill Book Co.: New York, NY, USA, 1997.

55. Upadhyay, S.K.; Singh, J.S.; Saxena, A.K.; Singh, D.P. Impact of PGPR inoculation on growth and antioxidant status of wheat under saline conditions. Plant Biol. 2012, 14, 605-611. [CrossRef] [PubMed]

56. Orhan, F. Alleviation of salt stress by halotolerant and halophilic plant growth promoting bacteria in wheat (Triticum aestivum). Braz. J. Microbiol. 2016, 47, 621-627. [CrossRef] [PubMed]

57. Gao, W.; Xu, F.-C.; Guo, D.-D.; Zhao, J.-R.; Liu, J.; Guo, Y.-W.; Singh, P.K.; Ma, X.-N.; Long, L.; Botella, J.R.; et al. Calcium-dependent protein kinases in cotton: Insights into early plant responses to salt stress. BMC Plant Biol. 2018, 18, 15. [CrossRef] [PubMed]

58. Yassin, A. Adverse effects of salinity on citrus. Int. J. Agric. Biol. 2005, 4, 668-680.

59. Muhammad, S.; Müller, T.; Joergensen, R.G. Relationships between soil biological and other soil properties in saline and alkaline arable soils from Pakistani Punjab. In Microbial Use of Organic Substrates and Maize Growth, Especially in Saline and Alkaline Soils of Pakistani Punjab. Ph.D. Thesis, Department of Soil Biology and Plant Nutrition, Faculty of Organic Agricultural Sciences, University of Kassel, Kassel, Germany, 2005; pp. $48-71$.

60. Diacono, M.; Montemurro, F. Effectiveness of organic wastes as fertilizers and amendments in salt-affected soils. Agriculture 2015, 5, 221-230. [CrossRef]

61. Garcia, C. Microbial activity in soils of SE Spain exposed to degradation and desertification processes. Strategies for their rehabilitation. In Research and Perspectives of Soil Enzymology in Spain; Garcia, C., Hernandez, M.T., Eds.; CEBASCSIC: Murcia, Spain, 2000; pp. 93-143.

62. Yousif, A.M.; Abdalla, M.A. Variations in nitrogen mineralization from different manures in semi-arid tropics of Sudan with reference to salt-affected soils. Int. J. Agric. Biol. 2009, 11, 515-520.

63. Munns, R.; Tester, M. Mechanism of salinity tolerance. Annu. Rev. Plant Biol. 2008, 59, 651-681. [CrossRef]

64. Shahzad, H.; Ullah, S.; Iqbal, M.; Bilal, H.M.; Shah, G.M.; Ahmad, S.; Zakir, A.; Ditta, A.; Farooqi, M.A.; Ahmad, I. Salinity types and level-based effects on the growth, physiology and nutrient contents of maize (Zea mays). Ital. J. Agron. 2019, 14, 199-207. [CrossRef]

65. Verma, G.; Sharma, R.P.; Sharma, S.P.; Subehia, S.K.; Shambhavi, S. Changes in soil fertility status of maize-wheat system due to long-term use of chemical fertilizers and amendments in an alfisol. Plant Soil Environ. 2012, 58, 529-533. [CrossRef]

66. Shaaban, M.; Abid, M.; Abou-Shanab, R.A.I. Amelioration of salt affected soils in rice paddy system by application of organic and inorganic amendments. Plant Soil Environ. 2013, 59, 227-233. [CrossRef]

67. Kamel, G.; Noufal, E.; Farid, I.; Abdel-Aziz, S.; Abbas, M. Alleviating salinity and sodicity by adding some soil amendments. J. Soil Sci. Agric. Eng. 2016, 7, 389-395.

68. Trivedi, P.; Singh, K.; Pankaj, U.; Verma, S.K.; Verma, R.K.; Patra, D.D. Effect of organic amendments and microbial application on sodic soil properties and growth of an aromatic crop. Ecol. Eng. 2017, 102, 127-136. [CrossRef]

69. Guo, X.; He, L.; Li, Q.; Yuan, D.; Deng, Y. Investigating the spatial variability of dissolved organic matter quantity and composition in Lake Wuliangsuhai. Ecol. Eng. 2014, 62, 93-101. [CrossRef]

70. Liu, H.; Khan, M.Y.; Carvalhais, L.C.; Delgado-Baquerizo, M.; Yan, L.; Crawford, M.; Dennis, P.G.; Singh, B.; Schenk, P.M. Soil amendments with ethylene precursor alleviate negative impacts of salinity on soil microbial properties and productivity. Sci. Rep. 2019, 9, 6892. [CrossRef] 
71. Wang, L.; Sun, X.; Li, S.; Zhang, T.; Zhang, W.; Zhai, P. Application of organic amendments to a coastal saline soil in north China: Effects on soil physical and chemical properties and tree growth. PLoS ONE 2014, 9, 89185. [CrossRef]

72. Abd El-Naby, Z.M.; Hafez, W.A.E.; Hashem, H.A. Remediation of salt-affected soil by natural and chemical amendments to improve berseem clover yield and nutritive quality. Afr. J. Range Forage Sci. 2019, 36, 49-60. [CrossRef]

73. Taffouo, V.D.; Wamba, O.F.; Yombi, E.; Nono, G.V.; Akoe, A. Growth, yield, water status and ionic distribution response of three bambara groundnut (Vigna subterranean L. verdc.) land races grown under saline conditions. Int. J. Bot. 2010, 6, 53-58.

74. Gong, X.L.; Liu, C.; Zhou, M.; Luo, L.Y.; Wang, L.; Wang, Y.; Hong, M.M.; Cai, J.W.; Gong, S.J.; Hong, F.S. Oxidative damages of maize seedlings caused by combined stress of potassium deficiency and salt stress. Plant Soil. 2011, 340, 443-452. [CrossRef]

75. Ozgur, R.; Uzilday, B.; Sekmen, A.H.; Turkan, I. Reactive oxygen species regulation and antioxidant defence in halophytes. Funct. Plant Biol. 2013, 40, 832-847. [CrossRef]

76. Uzilday, B.; Ozgur, R.; Sekmen, A.H.; Yildiztugay, E.; Turkan, I. Changes in the alternative electron sinks and antioxidant defense in chloroplasts of the extreme halophyte Eutrema parvulum (Thellungiella parvula) under salinity. Ann. Bot. 2015, 115, 449-463. [CrossRef] [PubMed]

77. Kaymakanova, M.; Stoeva, N. Physiological reaction of bean plants (Phaseolus vulgares L.) to salinity stress. Gen. Appl. Plant Physiol. 2008, 34, 177-188.

78. Kaymakanova, M.; Stoeva, N.; Mincheva, T. Salinity and its effect on physiological response of bean (Phaseolus vulgaris L.). Central. Eur. Agric. 2008, 9, 749-756.

79. Zafar, S.A.; Patil, S.; Uzair, M.; Fang, J.; Zhao, J.; Yuan, S.; Uzair, M.; Luo, Q.; Shi, J.; Schreiber, L.; et al. Degenerated panicle and partial sterility 1 (DPS1) encodes a CBS domain containing protein required for anther cuticle and panicle development in rice. New Phytol. 2019. [CrossRef]

80. Hasanuzzaman, M.; Nahar, K.; Gill, S.S.; Alharby, H.F.; Razafindrabe, B.H.; Fujita, M. Hydrogen peroxide pretreatment mitigates cadmium-induced oxidative stress in Brassica napus L.: An intrinsic study on antioxidant defense and glyoxalase systems. Front. Plant Sci. 2017, 8, 115. [CrossRef]

81. Ramzani, P.M.A.; Shan, L.; Anjum, S.; Ronggui, H.; Iqbal, M.; Virk, Z.A.; Kausar, S. Improved quinoa growth, physiological response, and seed nutritional quality in three soils having different stresses by the application of acidified biochar and compost. Plant Physiol. Biochem. 2017, 116, 127-138. [CrossRef]

82. Kamran, M.; Parveen, A.; Ahmar, S.; Malik, Z.; Hussain, S.; Chattha, M.S.; Chen, J.T. An Overview of Hazardous impacts of soil salinity in crops, tolerance mechanisms, and amelioration through Selenium supplementation. Int. J. Mole. Sci. 2020, 21, 148. [CrossRef]

83. Kamran, M.; Malik, Z.; Parveen, A.; Huang, L.; Riaz, M.; Bashir, S.; Mustafa, A.; Abbasi, G.H.; Xue, B.; Ali, U. Ameliorative effects of biochar on rapeseed (Brassica napus L.) growth and heavy metal immobilization in soil irrigated with untreated wastewater. J. Plant Growth Reg. 2019, 1-16. [CrossRef]

84. Kamran, M.; Malik, Z.; Parveen, A.; Zong, Y.; Abbasi, G.H.; Rafiq, M.T.; Shaaban, M.; Mustafa, A.; Bashir, S.; Rafay, M.; et al. Biochar alleviates Cd phytotoxicity by minimizing bioavailability and oxidative stress in pak choi (Brassica chinensis L.) cultivated in Cd-polluted soil. J. Environ. Manag. 2019, 250, 1-12. [CrossRef]

85. Naveed, M.; Mustafa, A.; Azhar, S.Q.; Kamran, M.; Zahir, Z.A.; Núñez-Delgado, A. Burkholderia phytofirmans $\mathrm{PsJN}$ and tree twigs derived biochar together retrieved $\mathrm{Pb}$-induced growth, physiological and biochemical disturbances by minimizing its uptake and translocation in mung bean (Vigna radiata L.). J. Environ. Manag. 2020, 257, 109974. [CrossRef]

86. Chaganti, V.N.; Crohn, D.M.; Sim_unek, J. Leaching and reclamation of a biochar and compost amended saline-sodic soil with moderate SAR reclaimed water. Agric. Water Manag. 2015, 158, 255e265. [CrossRef]

87. Hammer, E.C.; Forstreuter, M.; Rillig, M.C.; Kohler, J. Biochar increases arbuscular mycorrhizal plant growth enhancement and ameliorates salinity stress. Appl. Soil Ecol. 2015, 96, 114e121. [CrossRef]

88. Naveed, M.N.; Ramzan, A.; Mustafa, A.; Samad, B.; Niamat, M.; Yaseen, Z.; Ahmad, M.; Hasanuzzaman, N.; Sun, W.; Shi, X.M. Alleviation of salinity induced oxidative stress in Chenopodium quinoa by Fe biofortification and biochar-endophyte interaction. Agronomy 2020, 10, 168. [CrossRef]

89. Gao, S.; Ouyang, C.; Wang, S.; Xu, Y.; Tang, L.; Chen, F. Effects of salt stress on growth, antioxidant enzyme and phenylalanine ammonia-lyase activities in Jatropha curcas L. seedlings. Plant Soil Environ. 2008, 54, 374-381. [CrossRef] 
90. Abbasi, M.K.; Hina, M.; Khalique, A.; Khan, S.R. Mineralization of three organic manures used as nitrogen source in a soil incubated under laboratory conditions. Commun. Soil Sci. Plant Anal. 2007, 38, 1691-1711. [CrossRef]

91. Singh, J.S.; Pandey, V.C.; Singh, D.P.; Singh, R.P. Influence of pyrite and farmyard manure on population dynamics of soil methanotroph and rice yield in saline rain-fed paddy field. Agric. Ecosyst. Environ. 2010, 139, 74-79. [CrossRef]

92. Erdal, I.; Bozkurt, M.A.; Cimrin, K.M.; Karaca, S.; Salgam, M. Effects of humic acid and phosphorus fertilizer application on growth phosphorus uptake of maize (Zea mays L.) grown on a calcareous soil. Turk. J. Agric. Forest. 2000, 24, 663-668.

93. Taalab, A.S.; Hella, F.A.; Abou-Seeda, M.A. Influence of phosphate fertilizers enriched with sulfur on phosphorus availability and corn yield in calcareous soil in arid region. Ozean J. Appl. Sci. 2008, 1, 105-115.

94. Ditta, A.; Arshad, M.; Zahir, Z.A.; Jamil, A. Comparative Efficacy of Rock Phosphate Enriched Organic Fertilizer vs. Mineral Phosphatic Fertilizer for Nodulation, Growth and Yield of Lentil. Int. J. Agric. Biol. 2015, 17, 589-595. [CrossRef]

95. Qu, C.; Liu, C.; Gong, X.; Li, C.; Hong, M.; Wang, L.; Hong, F. Impairment of maize seedling photosynthesis caused by a combination of potassium deficiency and salt stress. Environ. Exp. Bot. 2012, 75, 134-141. [CrossRef]

96. Wong, V.N.L.; Dalal, R.C.; Greene, R.S.B. Carbon dynamics of sodic and saline soils following gypsum and organic material additions: A laboratory incubation. Appl. Soil Ecol. 2009, 41, 29-40. [CrossRef]

97. David, R.; Dimitrios, P. Diffusion and cation exchange during the reclamation of saline-structured soils. Geoderma 2002, 107, 271-279. [CrossRef]

98. Huang, X.; Jia, Z.; Guo, J.; Li, T.; Sun, D.; Meng, H.; Yu, G.; He, X.; Ran, W.; Zhang, S. Ten-year long-term organic fertilization enhances carbon sequestration and calcium-mediated stabilization of aggregate-associated organic carbon in a reclaimed Cambisol. Geoderma 2019, 355, 113880. [CrossRef]

99. Zhang, X.C.; Norton, L.D. Effect of exchangeable Mg on saturated hydraulic conductivity, disaggregation and clay dispersion of disturbed soils. J. Hydro. 2002, 260, 194-205. [CrossRef] 